\title{
Phosphate-Stabilized Lithium Intercalation Compounds
}

\author{
Thomas J. Richardson ${ }^{*}, z$ \\ Environmental Energy Technologies Division \\ Ernest Orlando Lawrence Berkeley National Laboratory \\ Berkeley, California 94720 USA
}

\begin{abstract}
Four manganese and iron phosphates with alluaudite or fillowite structures have been prepared by solid state reactions: $\mathrm{Na}_{2} \mathrm{FeMn}_{2}\left(\mathrm{PO}_{4}\right)_{3}, \mathrm{LiNaFeMn}_{2}\left(\mathrm{PO}_{4}\right)_{3}$, $\mathrm{NaFe}_{3}\left(\mathrm{PO}_{4}\right)_{3}$, and $\mathrm{Na}_{2} \mathrm{Mn}_{3}\left(\mathrm{PO}_{4}\right)_{3} . \mathrm{Li}_{\mathrm{x}} \mathrm{Na}_{2-\mathrm{x}} \mathrm{FeMn}_{2}\left(\mathrm{PO}_{4}\right)_{3}$ with $\mathrm{x}$ close to 2 was prepared from $\mathrm{Na}_{2} \mathrm{FeMn}_{2}\left(\mathrm{PO}_{4}\right)_{3}$ by molten salt ion exchange. These materials are similar in stoichiometry to the phospho-olivines $\mathrm{LiFe}(\mathrm{Mn}) \mathrm{PO}_{4}$, but have a more complex structure that can accommodate mixed transition metal oxidation states. They are of interest as candidates for lithium battery cathodes because of their somewhat higher electronic conductivity, high intercalant ion mobility, and ease of preparation. Their performance as intercalation electrodes in non-aqueous lithium cells was, however, poor.
\end{abstract}

\footnotetext{
*Electrochemical Society Active Member.

z E-mail: tjrichardson@lbl.gov
} 


\section{Introduction}

The lithium battery positive electrode couple $\mathrm{LiFePO}_{4} / \mathrm{FePO}_{4}$ has a theoretical capacity of $170 \mathrm{mAh} / \mathrm{g}$ with a flat discharge profile at $3.4 \mathrm{~V}$ vs. Li [1]. Despite its poor electronic conductivity, high utilization has been reported for carbon-coated electrodes at moderate discharge rates $[2,3]$. The strongly-bonded $\mathrm{PO}_{4}$ units enhance the thermal [4] and electrochemical [5] stability of $(\mathrm{Li}) \mathrm{FePO}_{4}$ toward oxygen loss and structural rearrangement. Because it is a two-phase system requiring movement of the phase boundary during charge/discharge [6], high rate cycling yields poor utilization. In addition, the synthesis of $\mathrm{LiFePO}_{4}$ must be carried out under low oxygen partial pressure, and the best electrode materials are obtained with long processing times $[7,8]$. The goal of the present work was to find improved cathode materials containing the inexpensive and low-toxicity metals iron or manganese. High electronic conductivity and ionic mobility, inherent stability, ease of preparation, and the ability to accommodate mixed transition metal oxidation states within a given phase were important criteria.

Among the wide variety of known iron and manganese phosphates are compounds with the alluaudite structure [9]. This group of compounds has the general formula $\mathrm{X}^{1} \mathrm{X}^{2} \mathrm{M}^{1} \mathrm{M}_{2}^{2}\left(\mathrm{PO}_{4}\right)_{3}$, where $\mathrm{X}^{1}$ and $\mathrm{X}^{2}$ are cations residing in different sites in caxis oriented tunnels formed by chains of edge-shared $\mathrm{MO}_{6}$ octahedra linked by tetrahedral $\mathrm{PO}_{4}$ units (Fig. 1). Hatert et al. [10] recently reported facile solid-state syntheses of $\mathrm{Li}_{\mathrm{X}} \mathrm{Na}_{1-\mathrm{x}} \mathrm{MnFe}_{2}\left(\mathrm{PO}_{4}\right)_{3}$ with $0<\mathrm{x}<0.9$, and showed that three additional $\mathrm{X}$ sites are available and that vacancies may occur on any of the five X positions. Mixed oxidation states are common for both $\mathrm{X}$ and $\mathrm{M}$ cations in both synthetic and naturallyoccurring alluaudites [11]. Other known alluaudites containing iron or manganese 
include $\mathrm{NaFe}_{3}\left(\mathrm{PO}_{4}\right)_{3}[12], \mathrm{NaFe}_{3.67}\left(\mathrm{PO}_{4}\right)_{3}[13], \mathrm{NaMn}_{3}\left(\mathrm{PO}_{4}\right)\left(\mathrm{HPO}_{4}\right)_{2}$ [14],

$\mathrm{Ag}_{2} \mathrm{FeMn}_{2}\left(\mathrm{PO}_{4}\right)_{3}$ [15], and $\mathrm{Cu}_{1.35} \mathrm{Fe}_{3}\left(\mathrm{PO}_{4}\right)_{3}$ [16]. The last exhibits good mixed conductivity [16] similar to that of the $\mathrm{NaSiCON} \mathrm{Na} 2.5 \mathrm{ZrFe}\left(\mathrm{PO}_{4}\right)_{3}$ [17].

\section{Experimental}

$\mathrm{Li}_{0.75} \mathrm{Na}_{0.25} \mathrm{MnFe}_{2}\left(\mathrm{PO}_{4}\right)_{3}, \mathrm{Na}_{2} \mathrm{FeMn}_{2}\left(\mathrm{PO}_{4}\right)_{3}, \mathrm{LiNaFeMn}\left(\mathrm{PO}_{4}\right)_{3}, \mathrm{NaFe}_{3}\left(\mathrm{PO}_{4}\right)_{3}$, and $\mathrm{Na}_{2} \mathrm{Mn}_{3}\left(\mathrm{PO}_{4}\right)_{3}$ were prepared by heating stoichiometric mixtures of $\mathrm{Li}_{2} \mathrm{CO}_{3}, \mathrm{Na}_{2} \mathrm{C}_{2} \mathrm{O}_{4}$, $\mathrm{MnCO}_{3}, \mathrm{FePO}_{4} \cdot 4 \mathrm{H}_{2} \mathrm{O}$, and $\mathrm{NH}_{4} \mathrm{H}_{2} \mathrm{PO}_{4}$ in air at $950^{\circ} \mathrm{C}$ in a $\mathrm{Pt}$ crucible for $16 \mathrm{~h}$, then quenching in air. $\mathrm{LiFePO}_{4}$ and $\mathrm{LiMnPO}_{4}$ were prepared by heating precursor mixtures in flowing dry $\mathrm{N}_{2}$ at $750^{\circ} \mathrm{C}$ for $48 \mathrm{~h}$. $\mathrm{Li}_{\mathrm{x}} \mathrm{Na}_{2-\mathrm{x}} \mathrm{FeMn}_{2}\left(\mathrm{PO}_{4}\right)_{3}$ was prepared by ion exchange from $\mathrm{Na}_{2} \mathrm{FeMn}_{2}\left(\mathrm{PO}_{4}\right)_{3}$ in a $10 \mathrm{LiNO}_{3} / \mathrm{KNO}_{3}$ melt at $245^{\circ} \mathrm{C}$. The products were examined by powder X-ray diffraction (XRD) and by transmission mode Fourier transform infrared spectroscopy (FTIR) in KBr pellets. Structures were refined by whole pattern fitting of XRD patterns using the program RIQAS (Materials Data, Inc.). Electrodes were prepared by mixing the powders with acetylene black $(10 \mathrm{w} / \mathrm{o})$ and polyvinylidene difluoride (5 w/o) in n-methylpyrrolidinone and casting the slurry onto stainless steel current collectors. Swagelok-type cells were assembled from $1.6 \mathrm{~cm}^{2}$ working electrodes, lithium counter electrodes, Celgard 3401 separators, and $1 \mathrm{M} \mathrm{LiPF}_{6} / \mathrm{EC}: \mathrm{DMC}$.

\section{Results and Discussion}

Element ratios and synthesis conditions are given in Table I along with results of XRD and FTIR characterization. Single-phase alluaudites were obtained for the compositions $\mathrm{Li}_{0.75} \mathrm{Na}_{0.25} \mathrm{MnFe}_{2}\left(\mathrm{PO}_{4}\right)_{3}, \mathrm{Na}_{2} \mathrm{FeMn}_{2}\left(\mathrm{PO}_{4}\right)_{3}$ (Figs. 1 and 2), and ion- 
exchanged $\mathrm{Li}_{\mathrm{x}} \mathrm{Na}_{2-\mathrm{x}} \mathrm{FeMn}{ }_{2}\left(\mathrm{PO}_{4}\right)_{3}$ (Fig. 3). In $\mathrm{Na}_{2} \mathrm{FeMn}_{2}\left(\mathrm{PO}_{4}\right)_{3}$, sodium ions occupy two sites in the tunnels, surrounded by eight oxide ions at mean distances of $2.52 \AA$ and $2.67 \AA$, respectively. Exchange of lithium for sodium was complete in the smaller site, with four short Li-O bond lengths of $2.27 \AA$ and $2.29 \AA$, while almost half the sodium remained in the larger site.

Samples prepared by melt-quenching reaction mixtures with the compositions $\mathrm{Li}_{1.0} \mathrm{Na}_{1.0} \mathrm{FeMn}_{2}\left(\mathrm{PO}_{4}\right)_{3}$ and $\mathrm{Li}_{1.5} \mathrm{Na}_{0.5} \mathrm{FeMn}_{2}\left(\mathrm{PO}_{4}\right)_{3}$ contained 15 and 30 w/o $\mathrm{LiMn}_{0.8} \mathrm{Fe}_{0.2} \mathrm{PO}_{4}[18]$ respectively. The alkali metal stoichiometries in the alluaudite phases (Table I) were estimated by difference. Melt-quenched $\mathrm{NaFe}_{3}\left(\mathrm{PO}_{4}\right)_{3}$, previously obtained only as a minor component by hydrothermal synthesis [12], was amorphous by XRD, but had an FTIR spectrum characteristic of alluaudite (Fig. 4). $\mathrm{Na}_{2} \mathrm{Mn}_{3}\left(\mathrm{PO}_{4}\right)_{3}$, on the other hand, adopted the more complex fillowite structure [19]. Reversible interconversion of alluaudites and fillowites has been reported [20].

The capacities for lithium intercalation and de-intercalation in these materials were surprisingly small (Fig. 5). While reproducible plateaus appear during discharging, the corresponding features during charging are less distinct and there is significant hysteresis. No major changes in the voltage profiles took place during cycling. The possibility of improving their performance through particle size reduction and carbon coating is being investigated.

\section{Conclusion}

Although these easily-prepared iron and manganese alluaudite and fillowite phosphates have electronic and structural properties that make them attractive cathode 
materials for lithium batteries, their reversible capacities appear to be very limited.

\section{Acknowledgment}

This work was supported by the Assistant Secretary for Energy Efficiency and Renewable Energy, Office of FreedomCAR and Vehicle Technologies of the U.S. Department of Energy under Contract No. DE-AC03-76SF00098. 


\section{References}

[1] A. K. Pahdi, K. S. Nanjundaswamy, J. B. Goodenough, J. Electrochem. Soc. 144 (1997) 1188.

[2] N. Ravet, Y. Chouinard, J. F. Magnan, S. Besner, M. Gauthier, M. Armand, J. Power Sources 97-98 (2001) 503.

[3] H. Huang, S.-C. Yin, L. F. Nazar, Electrochem. Solid-State Lett. 4 (2001) A170.

[4] A. S. Andersson, J. O. Thomas, B. Kalska, L.Häggström, Electrochem. Solid-State Lett. 3 (2000) 66.

[5] T. J. Richardson, 200th Meeting of The Electrochemical Society, San Francisco, CA, September, 2001.

[6] A. S. Anderson, J. O. Thomas, J. Power Sources 97-98 (2001) 498.

[7] A. Yamada, S. C. Chung, K. Hinomuka, J. Electrochem. Soc. 148 (2001) A224.

[8] M. Takahashi, S. Tobishima, K. Takei, Y. Sakurai, J. Power Sources 97-98 (2001) 508.

[9] P. B. Moore, Am. Mineral. 56 (1971) 1955.

[10] F. Hatert, P. Keller, F. Lissner, D. Antenucci, A.-M. Fransolet, Eur. J. Mineral. 12 (2000) 847.

[11] R. P. Hermann, F. Hatert, A.-M. Fransolet, G. J. Long, F. Grandjean, Solid State Sci. 4 (2002) 507.

[12] D. R. Corbin, J. F. Whitney, W. C. Fultz, G. D. Stucky, M. M. Eddy, A. K. Cheetham, Inorg. Chem. 25 (1986) 2279.

[13] M. B. Korzenski, G. L. Schimek, J. W. Kolis, G. J. Long, J. Solid State Chem. 139 (1998) 152. 
[14] F. Leroux, A. Mar, C. Payen, D. Guyomard, A. Verbaere, Y. Piffard, J. Solid State Chem. 115 (1995) 240.

[15] N. Chouaibi, A. Daidouh, C. Pico, A. Santrich, M. L. Veiga, J. Solid State Chem. $159(2001) 46$.

[16] T. E. Warner, W. Milius, J. Maier, Solid State Ionics, 74 (1994) 119.

[17] O. Tillement, J. Angenault, J. C. Couturier, M. Quarton, Solid State Ionics, 44 (1991) 299.

[18] A. Yamada, S.-C. Chung, J. Electrochem. Soc. 148 (2001) A960.

[19] T. Araki, P. B. Moore, Am. Mineral. 66 (1981) 827.

[20] D. Antenucci, P. Tarte, A.-M. Fransolet, N. Jb. Miner. Mh. 1996 (1996) 289. 
Table I. Synthetic parameters and analytical data.

\begin{tabular}{|c|c|c|c|c|c|c|c|c|c|c|c|c|c|}
\hline $\mathrm{Li}$ & $\mathrm{Na}$ & $\mathrm{Fe}$ & $\mathrm{Mn}$ & $\begin{array}{l}\text { Temp } \\
\left({ }^{\circ} \mathrm{C}\right)\end{array}$ & Color & XRD & FTIR $^{\mathrm{a}}$ & $\mathrm{a}(\AA)$ & $\mathrm{b}(\AA)$ & $\mathrm{c}(\AA)$ & $\operatorname{beta}\left(^{\circ}\right)$ & $\begin{array}{l}\text { Vol } \\
\left(\AA^{3}\right)\end{array}$ & $\begin{array}{r}\mathrm{Vol} / \mathrm{Z} \\
\left(\AA^{3}\right)\end{array}$ \\
\hline & 1 & 3 & & 950 & Dk. Brown & Amorphous & A & & & & & & \\
\hline & 2 & 3 & & 960 & Dk. Brown & $\mathrm{Na}_{3} \mathrm{Fe}_{2}\left(\mathrm{PO}_{4}\right)_{3}$ & & & & & & & \\
\hline & 1 & 2 & 1 & $950^{\mathrm{b}}$ & & & & 12.001 & 12.538 & 6.405 & 114.45 & 877.3 & 219.3 \\
\hline 0.75 & 0.25 & 2 & 1 & 960 & Olive & Alluaudite & A & 11.970 & 12.472 & 6.388 & 114.71 & 866.3 & 216.6 \\
\hline & 2 & 1 & 2 & 900 & Rust & Alluaudite & A & 12.037 & 12.609 & 6.498 & 114.48 & 897.6 & 224.4 \\
\hline 0.6 & 1.2 & 1 & 2 & 950 & Rust & Alluaudite ${ }^{c}$ & A & 12.027 & 12.570 & 6.460 & 114.78 & 886.7 & 220.3 \\
\hline 0.9 & 0.7 & 1 & 2 & 950 & Red & Alluaudite $^{\mathrm{d}}$ & A & 12.024 & 12.546 & 6.440 & 114.89 & 881.3 & 221.7 \\
\hline 1.8 & 0.2 & 1 & 2 & $245^{\mathrm{e}}$ & Dk. Brown & Alluaudite & A & 12.002 & 12.540 & 6.468 & 115.17 & 881.0 & 220.3 \\
\hline & 1 & & 3 & 900 & Lt. Brown & $\mathrm{Mn}_{2} \mathrm{P}_{2} \mathrm{O}_{7}$ & & & & & & & \\
\hline & 2 & & 3 & 900 & Lavender & Fillowite & & 15.302 & & 43.696 & & & 211.0 \\
\hline \multicolumn{14}{|c|}{${ }^{\mathrm{a}} \mathrm{A}=$ alluaudite spectrum. } \\
\hline \multicolumn{14}{|c|}{${ }^{\mathrm{b}}$ Data from Ref. 10.} \\
\hline \multicolumn{14}{|c|}{${ }^{\mathrm{c}}+15^{\mathrm{w}} / \mathrm{o} \mathrm{LiMn}_{0.8} \mathrm{Fe}_{0.2} \mathrm{PO}_{4} ;$ from reaction mixture with $1: 1 \mathrm{Li}: \mathrm{Na}$ ratio. } \\
\hline \multicolumn{14}{|c|}{${ }^{\mathrm{d}}+30 \mathrm{w} / \mathrm{L} \mathrm{LiMn}_{0.8} \mathrm{Fe}_{0.2} \mathrm{PO}_{4} ;$ from reaction mixture with 1.5:0.5 Li:Na ratio. } \\
\hline & ${ }^{\mathrm{e}} \mathrm{pr}$ & & ad hy & ion-oyc & hange from & ${ }_{2} \mathrm{FeMn}_{2}\left(\mathrm{PO}_{4}\right)_{3}$. & & & & & & & \\
\hline
\end{tabular}




\section{Figure Captions}

1. $\mathrm{Na}_{2} \mathrm{FeMn}_{2}\left(\mathrm{PO}_{4}\right)_{3}$ alluaudite structure.

2. Observed and refined XRD patterns for $\mathrm{Na}_{2} \mathrm{FeMn}_{2}\left(\mathrm{PO}_{4}\right)_{3}$.

3. Observed and refined XRD patterns for $\mathrm{Li}_{\mathrm{X}} \mathrm{Na}_{2-\mathrm{x}} \mathrm{FeMn}_{2}\left(\mathrm{PO}_{4}\right)_{3}$.

4. FTIR spectra of a) $\mathrm{Na}_{2} \mathrm{FeMn}_{2}\left(\mathrm{PO}_{4}\right)_{3}$, b) $\left.\mathrm{Na}_{2} \mathrm{MnFe}_{2}\left(\mathrm{PO}_{4}\right)_{3}, \mathrm{c}\right) \mathrm{NaFe}_{3}\left(\mathrm{PO}_{4}\right)_{3}$.

5. Charge/discharge profiles for a) $\mathrm{NaFe}_{3}\left(\mathrm{PO}_{4}\right)_{3}$ and b) $\mathrm{Li}_{\mathrm{x}} \mathrm{Na}_{2-\mathrm{x}} \mathrm{FeMn}_{2}\left(\mathrm{PO}_{4}\right)_{3}$ in lithium cells. 


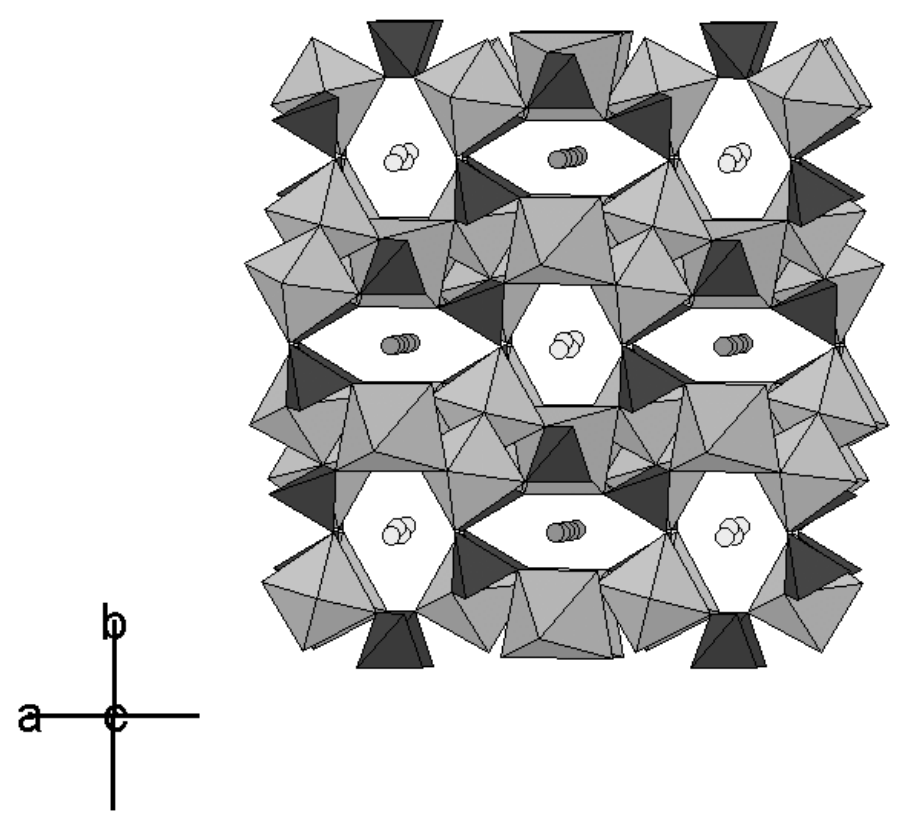

Richardson, Fig. 1 


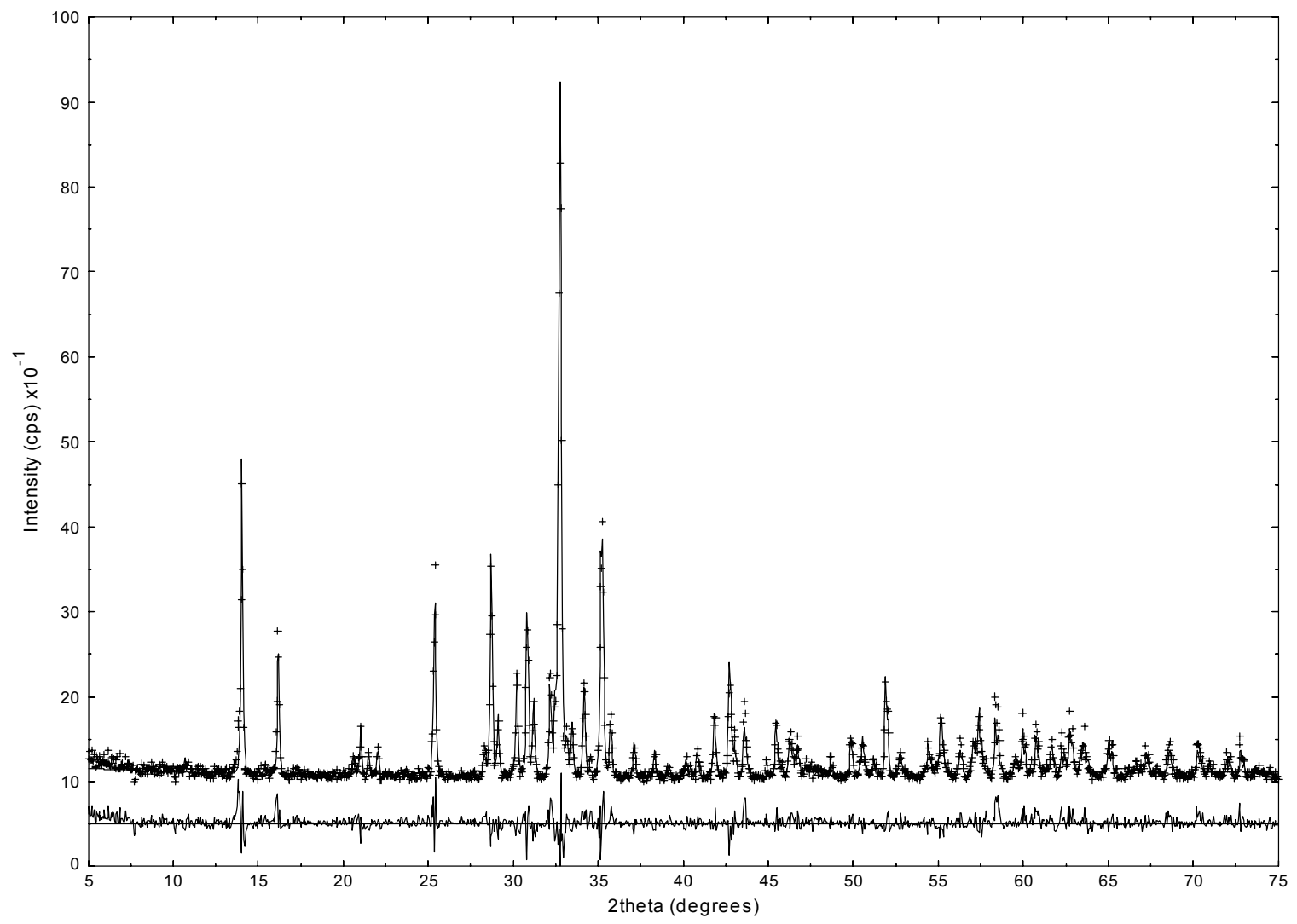

Richardson, Fig. 2 


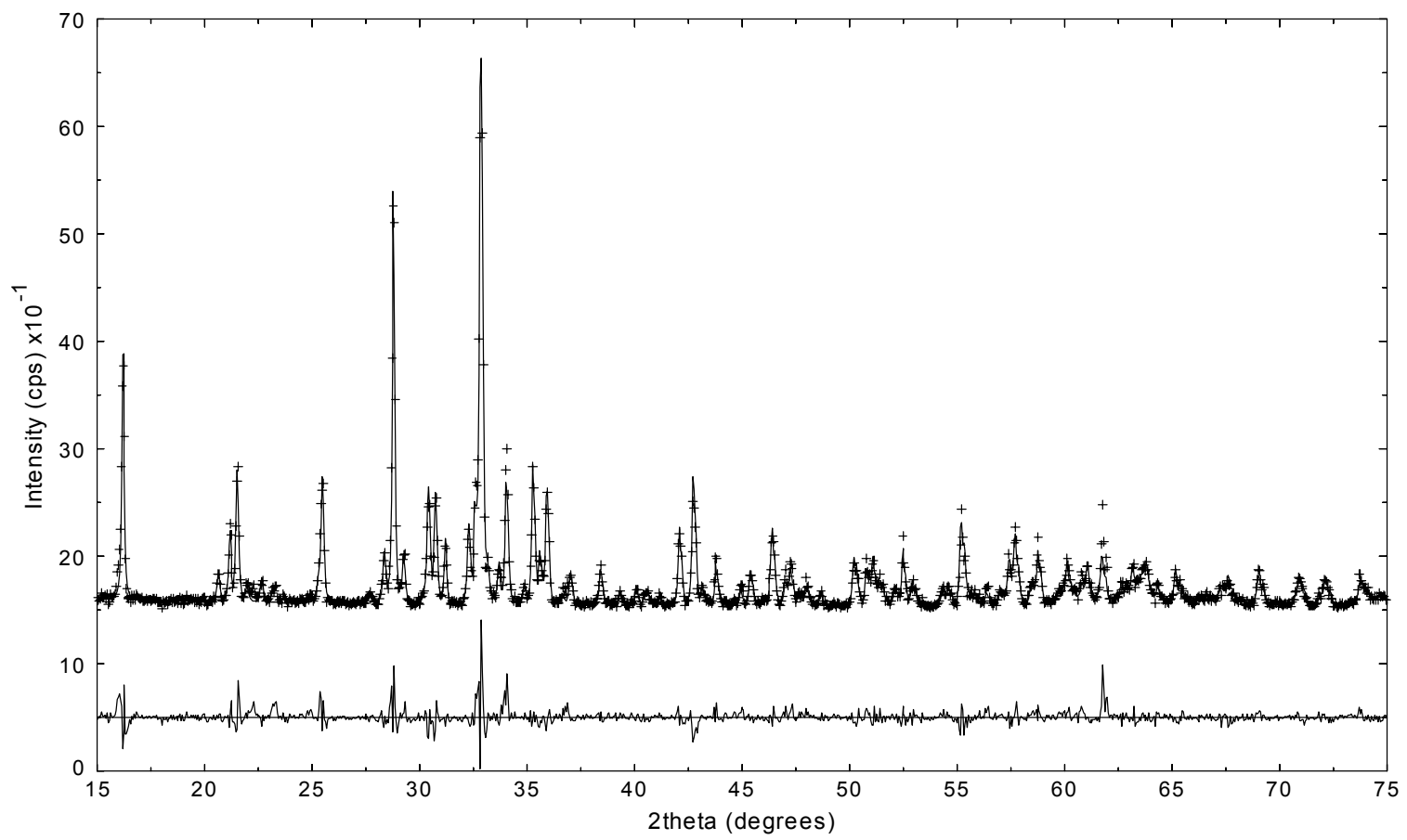

Richardson, Fig. 3 


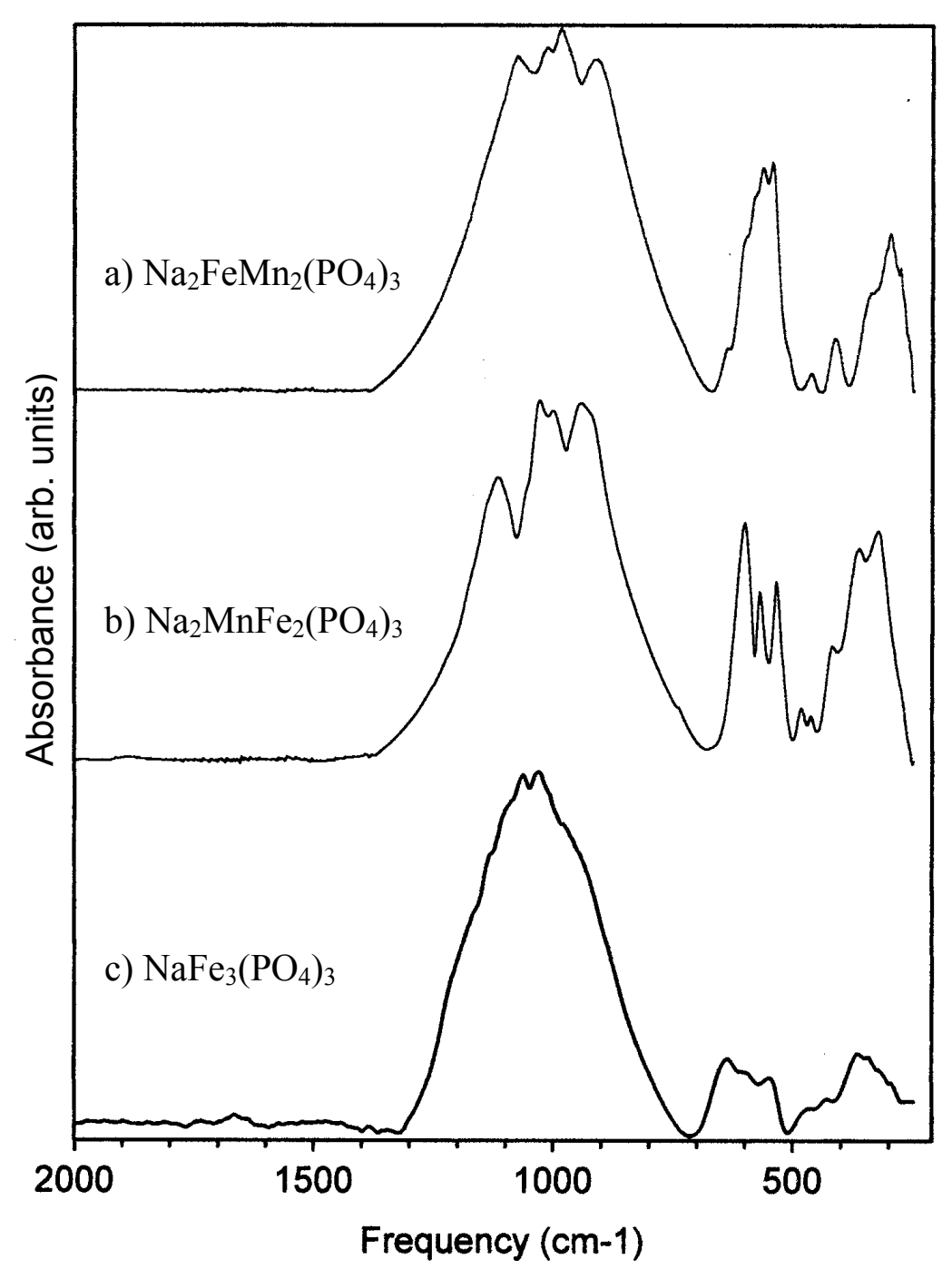

Richardson, Fig. 4 

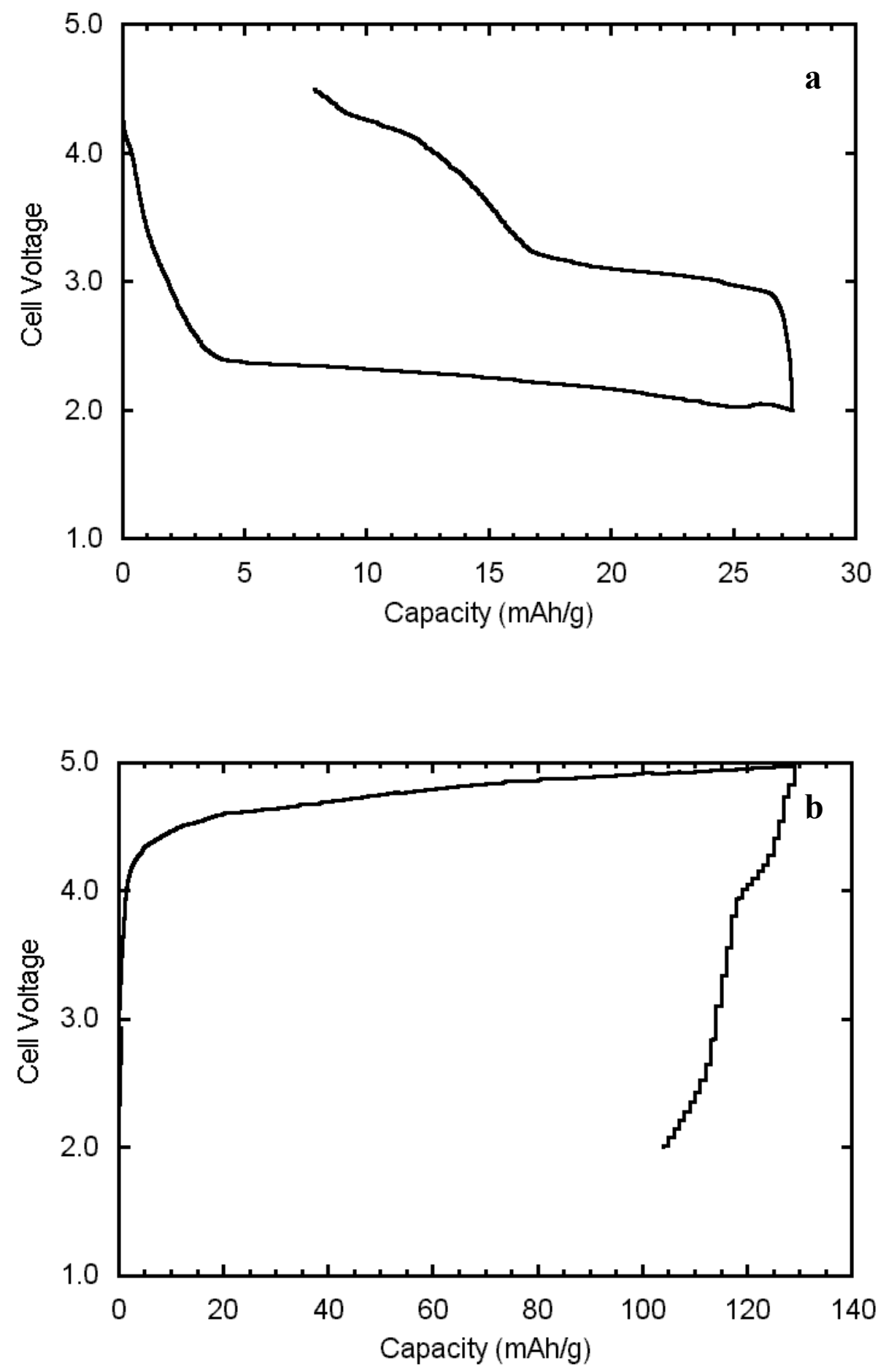

Richardson, Fig. 5 